
\section{Ecitus} Hypertension Service, Hammersmith Hospital, Imperial College Healthcare NHS Trust, London, UK ${ }^{2}$ Department of Respiratory Medicine, Northern General Hospital, Sheffield, UK

\section{Correspondence to} Dr Luke S Howard, National Pulmonary Hypertension Service, Hammersmith Hospital, Imperial College Healthcare NHS Trust, Du Cane Road, London W12 OHS, UK:

l.howard@imperial.ac.uk

Received 6 November 2012 Revised 6 November 2012 Accepted 16 November 2012 Published Online First 12 December 2012

\title{
NICE guideline: management of venous thromboembolic diseases and role of thrombophilia testing
}

\author{
Luke S Howard, ${ }^{1}$ Rodney J Hughes ${ }^{2}$
}

\section{ABSTRACT}

The National Institute for Health and Clinical Excellence recently published a clinical guideline on the management of venous thromboembolic disease and thrombophilia testing. Several stand-out

recommendations are made which may be practice changing for many physicians, such as catheter-directed thrombolysis for ilio-femoral deep venous thrombosis, routine cancer screening and extended duration of anticoagulation for unprovoked events. In this article, we summarise the key points of the guideline and discuss remaining areas of controversy.

\section{INTRODUCTION}

In June 2012 the National Institute for Health and Clinical Excellence (NICE) published clinical guideline 144 on the management of venous thromboembolic diseases (VTE) and the role of thrombophilia. ${ }^{1}$ This superceded the update planned by the British Thoracic Society (BTS) of the pulmonary embolism guideline, last updated in 2003. ${ }^{2}$ The NICE document covers diagnosis of suspected pulmonary embolus (PE) and deep venous thrombosis (DVT) and subsequent acute management, but excludes children and pregnancy. While it does elaborate on the role of thrombophilia testing, it provides no guidance on how to weigh up the risks and benefits of prolonged anticoagulation.

The NICE guideline differs from what readers may normally expect to see in VTE guidelines drafted by national or international bodies, such as those previously produced by the British Thoracic Society, ${ }^{2}$ European Society of Cardiology (ESC) ${ }^{3}$ or American College of Chest Physicians (ACCP) ${ }^{4}$ in that the committee assessed both clinical and cost effectiveness before reaching a recommendation. When there is no evidence for review, no recommendations, for example on the basis of expert opinion, are made, which leaves some areas uncovered and unlicensed indications for novel therapies are also omitted. Furthermore, certain aspects of management, such as risk stratification, follow-up and home treatment are not covered.

The purpose of this article is not to provide a comprehensive summary of the guideline, rather to provide core recommendations and update the reader on new recommendations, while providing the rationale of the NICE guideline development group (GDG). Readers who wish to read a fuller set of recommendations should also refer to the latest $\mathrm{ESC}^{3}$ and ACCP guidelines. ${ }^{4}$

The full version of the guideline document with shorter summaries can be found at http://guidance. nice.org.uk/CG144.

\section{NEW RECOMMENDATIONS Diagnosis}

The GDG recommended moving from a three-way (low, medium and high probability) to two-way Wells score for DVT and PE and if these are deemed likely then proceeding to diagnostic imaging, with compression Doppler ultrasound of the leg veins and CT pulmonary angiography (CTPA), respectively, as the modality of choice. For DVT, if the first ultrasound scan is negative, then further risk stratification with d-dimer should be undertaken, and if positive, the patient re-scanned. When there is concern about the risk of radiation, contrast allergy or renal impairment, nuclear medicine ventilationperfusion (VQ) scanning is an alternative, preferably using single photon emission CT (SPECT), but planar if not available. When CTPA or VQ is negative and a DVT is suspected, further imaging of the leg veins with ultrasound is recommended. Ultrasound of the leg veins is not recommended as a first-line imaging investigation in patients presenting with symptoms and/or signs of PE and DVT. In the presence of a low likelihood of DVT or PE using the Wells score, then d-dimer stratification is advised. Positive assays necessitate further imaging, whereas an alternative diagnosis should be sought in patients with negative assays. Previous guidelines ${ }^{2} 3$ have advocated the use of echocardiography to diagnose PE when the patient's condition is unstable and rapid intervention is required. This issue is not covered by the GDG.

\section{Treatment}

Patients should be offered treatment if imaging is not available immediately in the case of PE or within $4 \mathrm{~h}$ for suspected DVT. Once the diagnosis has been confirmed then anticoagulation should be commenced with subcutaneous low molecular weight heparin (LMWH) or fondaparinux for at least 5 days or until an international normalised ratio (INR) of $>2$ is achieved with a vitamin $\mathrm{K}$ antagonist (VKA). In the presence of renal impairment (estimated glomerular filtration rate $<30 \mathrm{ml} / \mathrm{min} / 1.73 \mathrm{~m}^{2}$ ), either unfractionated heparin or LMWH with anti-factor Xa monitoring should be used and when there is a risk of bleeding, unfractionated heparin is advised. Patients 
with active malignancy should receive LMWH (see section below for duration).

One of the more stand-out recommendations is the advice to perform catheter-directed thrombolysis for symptomatic iliofemoral DVT when it has been present for up to 14 days in patients with good function, low risk of bleeding and a life expectancy of more than 1 year. The principal driving factor for this recommendation was from studies of systemic and catheterdirected thrombolysis showing a reduction in post-thrombotic syndrome (PTS), with a lower risk of bleeding with catheterdirected versus systemic administration, although it was deemed low-quality evidence.

In recent guidelines from learned societies, ${ }^{2}{ }^{3} \mathrm{PE}$ has been risk stratified into three categories, low, medium or high, or non-massive, sub-massive and massive PE. However, the GDG felt that there was insufficient evidence to justify different treatment strategies for these three groups and thus suggested only classifying patients as having haemodynamic stability or instability, as defined by systolic blood pressure $<90 \mathrm{~mm} \mathrm{Hg}$ or a pressure drop of $\geq 40 \mathrm{~mm} \mathrm{Hg}$ for $>15 \mathrm{~min}$ if not caused by an arrhythmia, hypovolaemia or sepsis. A positive recommendation was made for the use of unfractionated heparin and subsequent systemic thrombolysis for the treatment of haemodynamically unstable PE, but not catheter-directed thrombolysis. However, the GDG recognised that there was a potentially important increased risk of major bleeding. There was insufficient evidence to recommend thrombolysis to stable patients, such as those with documented right ventricular dysfunction.

When there is a contraindication to anticoagulation or thrombolysis due to bleeding risk, inferior vena cava filter placement is recommended, with a plan for removal once anticoagulation can safely commence, but no recommendation was given regarding the role of mechanical clot disruption or surgical embolectomy, in the absence of adequate data to review. Filter insertion was also suggested when recurrent PE is diagnosed despite anticoagulation with an increased INR target range of 3-4 or the use of LMWH.

Lastly, for patients with a diagnosis of DVT, below knee ipsilateral graduated compression stockings $(>23 \mathrm{~mm} \mathrm{Hg})$ are recommended for the prevention of post-thrombotic syndrome, at least 1 week after treatment has begun or once leg swelling has subsided. Patients should continue to use these for 2 years and they should be re-fitted twice or three times a year.

\section{Duration of treatment and role of thrombophilia testing}

For patients with a clear temporary provoking risk factor for VTE, anticoagulation is recommended for a period of 3 months. If the patient has active cancer, they should receive treatment with LMWH for 6 months before reviewing the decision to continue anticoagulation with a VKA. For unprovoked events, a decision whether to continue or discontinue anticoagulation should be made at 3 months. This decision should be taken after discussion with the patient, having taken in to account the long-term risk of bleeding and risk of recurrence. The GDG felt that the recommendation for long-term anticoagulation following PE was stronger than following DVT, since the risk of the recurrent event being a $\mathrm{PE}$, and thus carrying greater morbidity and mortality, is greater if the primary event is a PE. The complexity of this decision-making process usually requires it to be made in the secondary care setting. The decision should then be reviewed annually since relative risks and circumstances may change. No guidance is provided on how to assess this balance, since it was felt by the GDG that no rule of thumb or validated tool exists to assess risk.
In recognising that the presence of thrombophilia does not predict the risk of recurrence following an unprovoked episode of VTE, the GDG were mindful to reduce the rate of unnecessary thrombophilia investigations and provide clear guidance on when it may impact on treatment decisions. As such, the only clear indications to perform thrombophilia testing were when consideration was being given to discontinuing anticoagulation following an unprovoked DVT or PE. Patients with a history of a first-degree relative with VTE should undergo testing for hereditary thrombophilia, but testing should not be extended to relatives, and all patients should undergo testing for antiphospholipid antibodies (detected as a lupus anticoagulant or as antibodies against cardiolipin or $\beta 2$-glycoprotein I).

Lastly, the GDG stressed the importance of patient information and education relating to lifestyle issues while on anticoagulation, when receiving dental treatment and during pregnancy.

\section{Investigations for cancer}

This guideline for the first time recommends routine screening for cancer in patients over the age of 40 following an unprovoked VTE episode. This was based on a single study which showed no statistically significant reduction in cancer-related mortality, but the GDG felt that the non-significant reduction was potentially clinically and economically important, with patients and relatives also being reassured by negative investigations. Despite the lack of good evidence for a reduction in cancer-related mortality, the screening strategy used in the study showed an increased pick-up of cancers (in approximately 1 in 10 patients with 93\% sensitivity) and at an earlier stage than in the standard care group. The study used a strategy of abdominal/pelvic CT or abdominal/pelvic ultrasound scan, alone or in combination with other tests (mammography, sputum cytology, tumour markers, faecal occult bloods and colonoscopy). The most cost-effective strategy, however, appeared to be abdominal/ pelvic CT, mammography and sputum cytology on top of the routine screening using history/examination, chest radiograph, blood tests (including serum calcium and liver function) and urinalysis, all at the time of diagnosis. Sputum cytology was removed from the final recommendation due to its limited role in the diagnosis of lung cancer. The impact of additional radiation was not taken into consideration, since the increased risk of cancer was felt to be very small compared with the increased early detection of cancer.

\section{AREAS FOR RESEARCH}

Many areas for research were identified by the GDG, but the following five areas were highlighted as being of high importance:

- The value of whole leg versus proximal leg vein ultrasound in the diagnosis of acute DVT.

- Long-term versus 3-month anticoagulation in patients with a high risk of recurrence.

- Treating with LMWH versus VKA beyond 6 months in patients with VTE in association with active cancer.

- The clinical and cost effectiveness of catheter-directed thrombolytic therapy or pharmacomechanical thrombolysis for the removal of acute proximal DVT versus standard anticoagulation.

- Systemic pharmacological thrombolysis versus standard anticoagulation for the treatment of acute PE with right ventricular dysfunction. 


\section{ISSUES NOT COVERED}

Certain areas of VTE management were explicitly excluded from the guideline, such as children (aged <18) and women who are pregnant. While recent guidance for these populations has been published by other organisations, ${ }^{4-6}$ several areas pertaining to the management of PE in particular are left open by the NICE guideline:

- The role of the new oral direct thrombin and anti-Xa inhibitor anticoagulant agents are not covered, and for information on these, the reader is referred to the ACCP guideline published earlier this year ${ }^{4}$ and the subsequent technology appraisal for rivaroxaban in the treatment of DVT and prevention of recurrent VTE. ${ }^{8}$

- The guideline does not cover risk stratification beyond defining haemodynamic instability for the purposes of consideration of thrombolysis and thus no recommendations are made on who may be considered eligible for home treatment of VTE. This is despite the availability of well validated clinical risk assessment scores, such as the Pulmonary Embolism Severity Index (PESI) score, which allow stratification of immediate risk following PE (30-day mortality). ${ }^{9}$ This score clearly defines a low-risk population potentially suitable for home management. ${ }^{10}$ PESI may also be useful to guide physicians in further evaluating haemodynamically stable patients and indentifying who remains at high risk of death, ${ }^{11}$ even if there can be no clear guidance on which of these patients may benefit from thrombolysis.

- No advice is given on how to follow up patients with VTE with respect to screening for complications, such as postthrombotic syndrome or chronic thromboembolic disease. This is despite guidance from the British Thoracic Society suggesting follow up at 3-6 months following PE. ${ }^{12}$

- Also, as outlined above, no guidance is provided on how to balance risk of recurrence versus risk of bleeding in determining whether to continue anticoagulation, and the reader should be referred again to the ACCP guideline. ${ }^{7}$

\section{IMPLICATIONS FOR SERVICES/RESOURCES}

This guideline considered the economic impact of the recommendations and highlights some of the implications for services. In our view, the principal impact of this guideline will be on diagnostic services in trying to move over to VQ SPECT from planar imaging and covering the increased demand for cancer screening, and the increased demand from vascular surgical departments or interventional radiology to perform catheterdirected thrombolysis for ilio-femoral DVT.

The recommendation to consider CT imaging of the abdomen and pelvis in patients with idiopathic VTE is likely to generate a significant increase in requests for such assessments, although the evidence for this approach is still somewhat controversial and not recommended in other guidance. ${ }^{3}$

Additionally the recent development in England and Wales of a tariff-based payment for the ambulatory management of PE is now left without any standardised assessment of suitability. It is left to individual organisations to determine the criteria by which this approach can be applied.

\section{CONCLUSION}

NICE clinical guideline 144 standardises the initial investigation and management of VTE and provides recommendations for the type and duration of anticoagulation based on well established clinical criteria. Several important areas, however, are not addressed and for the physician attending the patient, this guideline leaves some areas of clinical uncertainty in the management of acute PE. We recommend that readers refer to other documents for top-up guidance. ${ }^{3-6}$

Contributors $\mathrm{LH}$ produced the first draft of the manuscript. It was subsequently reviewed, edited and supplemented by $\mathrm{RH}$.

Funding None.

Competing interests $\mathrm{LH}$ has received consultancy fees and research funding from Bayer PLC. RH has received consultancy and lecture fees and travel grants from Sanofi Aventis, GlaxoSmithKline, Pfizer and Novartis.

Provenance and peer review Commissioned; internally peer reviewed.

\section{REFERENCES}

1 NICE. Venous Thromboembolic Diseases: The Management of Venous Thromboembolic Diseases and the Role of Thrombophilia Testing. Clinical guideline 144. London: National Institute for Health and Clinical Excellence, 2012.

2 British Thoracic Society Standards of Care Committee Pulmonary Embolism Guideline Development Group. British Thoracic Society guidelines for the management of suspected acute pulmonary embolism. Thorax 2003;58:470-83.

3 Torbicki A, Perrier A, Konstantinides S, Agnelli G, Galie N, Pruszczyk P, et al. Guidelines on the diagnosis and management of acute pulmonary embolism: the Task Force for the Diagnosis and Management of Acute Pulmonary Embolism of the European Society of Cardiology (ESC). Eur Heart J 2008;29:2276-315.

4 Guyatt GH, Akl EA, Crowther M, Gutterman DD, Schuunemann HJ. Executive summary: Antithrombotic Therapy and Prevention of Thrombosis, 9th ed: American College of Chest Physicians Evidence-Based Clinical Practice Guidelines. Chest 2012;141(2 Suppl):7S-47S

5 Royal College of Obstetricians and Gynaecologists. The Acute Management of Thrombosis and Embolism During Pregnancy and the Puerperium. 2007.

6 Chalmers E, Ganesen V, Liesner R, Maroo S, Nokes T, Saunders D, et al. Guideline on the investigation, management and prevention of venous thrombosis in children. Br J Haematol 2011;154:196-207.

7 Kearon C, Akl EA, Comerota AJ, Prandoni P, Bounameaux H, Goldhaber SZ, et al. Antithrombotic therapy for VTE disease: Antithrombotic Therapy and Prevention of Thrombosis, 9th ed: American College of Chest Physicians Evidence-Based Clinical Practice Guidelines. Chest 2012;141(2 Suppl):e419S-94S.

8 NICE. Rivaroxaban for the Treatment of Deep Vein Thrombosis and Prevention of Recurrent Deep Vein Thrombosis and Pulmonary Embolism. London: National Institute for Health and Clinical Excellence, 2012.

9 Aujesky D, Obrosky DS, Stone RA, Auble TE, Perrier A, Cornuz J, et al. Derivation and validation of a prognostic model for pulmonary embolism. Am J Respir Crit Care Med 2005;172:1041-6.

10 Aujesky D, Roy PM, Verschuren F, Righini M, Osterwalder J, Egloff M, et al. Outpatient versus inpatient treatment for patients with acute pulmonary embolism: an international, randomised non-inferiority trial. Lancet 2011;378:41-8.

11 Jimenez D, Aujesky D, Moores L, Gomez V, Marti D, Briongos S, et al. Combinations of prognostic tools for identification of high-risk normotensive patients with acute symptomatic pulmonary embolism. Thorax 2011;66:75-81.

12 British Thoracic Society Standards of Care Committee. BTS statement on criteria for specialist referral, admission, discharge and follow-up for adults with respiratory disease. Thorax 2008;63(Suppl 1):i1-16. 